\title{
RACK1 affects glioma cell growth and differentiation through the CNTN2-mediated RTK/Ras/MAPK pathway
}

\author{
YU YAN and YUGANG JIANG
}

Department of Neurosurgery, The Second Xiangya Hospital of Central South University, Changsha, Hunan 410011, P.R. China

Received June 23, 2015; Accepted November 11, 2015

DOI: $10.3892 / \mathrm{ijmm} .2015 .2421$

\begin{abstract}
Receptor for activated C kinase 1 (RACK1) and contactin-2 (CNTN2) are known to be abnormally expressed in gliomas; however, the association between RACK1 and CNTN2, and the effects of RACK1 and CNTN2 on glioma cell differentiation and the related molecular mechanisms remain largely unknown. The present study aimed to investigate the interaction between RACK1 and CNTN2, and to examine whether RACK1/CNTN2/receptor tyrosine kinase (RTK)/Ras/ mitogen-activated protein kinase (MAPK) axis plays a role in glioma growth and differentiation. The results from western blot analysis revealed that the protein expression levels of RACK1 and CNTN2 were higher in high-grade glioma tissues and cells, and lower in low-grade glioma tissues and cells. A co-immunoprecipitation assay demonstrated that RACK1 interacts with CNTN2, and RACK1 upregulated the expression of CNTN2. Gain-of-function and loss-of-function experiments indicated that both RACK1 and CNTN2 promoted glioma cell proliferation, inhibited glioma cell differentiation and activated the RTK/Ras/MAPK pathway. However, the effects of RACK1 on glioma cell proliferation, differentiation and the activation of the RTK/Ras/MAPK signaling pathway were abolished by the knockdown of CNTN2 using siRNA. In Therefore, the findings of this study firstly demonstrate that RACK1 interacts with CNTN2, and that the effects of RACK1 on glioma cell growth and differentiation are mediated by CNTN2. The RACK1/CNTN2/RTK/Ras/MAPK axis exists in glioma cells, and it may be a potential therapeutic target in gliomas.
\end{abstract}

\section{Introduction}

Gliomas are the most common primary brain tumors in humans. Malignant gliomas are highly aggressive. Although the standard treatment for malignant gliomas has evolved over

Correspondence to: Professor Yugang Jiang, Department of Neurosurgery, The Second Xiangya Hospital of Central South University, 139 Renmin Road, Changsha, Hunan 410011, P.R. China E-mail: yugangjiang1025@126.com

Key words: receptor for activated C kinase 1, contactin-2, differentiation, receptor tyrosine kinase/Ras/mitogen-activated protein kinase pathway the past 30 years, the average life expectancy for patients with malignant gliomas is only 14 months (1). With the development of molecular biology, previous research has suggested gene therapy strategies for the treatment of gliomas (2-6).

The receptor for activated $\mathrm{C}$ kinase 1 (RACK1) is a versatile scaffold protein involved in the regulation of a variety of cellular processes, including cell growth, migration, survival and metastasis (7-9). Previous studies have indicated that RACK1 is abnormally expressed in various malignant tumors, and its dysregulation may contribute to tumorigenesis (10-13). Certain pathways have been demonstrated to be activated by RACK1, such as the Src/Akt signaling pathway, mitogen-activated protein kinase (MAPK) signaling pathway, and the sonic hedgehog signaling pathway (14-16). RACK1 is widely expressed in the central nervous system, and it has been suggested that RACK1 is associated with the development of gliomas (14).

Contactin-2 (also known as CNTN2 or axonal glycoprotein TAG-1) protein is a member of the immunoglobulin superfamily. It functions as a cell adhesion molecule and is involved in the development of a variety of tumors $(17,18)$. Rickman et al reported that CNTN2 is overexpressed in gliomas (19). CNTN2 is the hub node in the protein-protein interaction network of oligodendroglioma, and it may serve an essential function in the pathogenesis of oligodendroglioma (20). It is thus of interest to identify the proteins that interact with CNTN2 in order to mediate its role in tumor development.

RACK1 is a scaffold protein that physically or functionally interacts with numerous proteins $(8,21-23)$, and CNTN2 has been linked to the highest degree in the oligodendroglioma proteinprotein interaction network (20). However, to the best of our knowledge, the interaction between RACK1 and CNTN2 has not been reported to date. In the present study, we firstly examined the association between RACK1 and CNTN2 in glioma cells. Certain studies have demonstrated that the receptor tyrosine kinase (RTK)/Ras/extracellular signal-regulated kinase (ERK) MAPK signaling pathway is associated with the growth and progression of gliomas $(24,25)$. Furthermore, we examined the possibility that RACK1/CNTN2/RTK/Ras/MAPK axis plays a role in glioma cell growth and differentiation.

\section{Materials and methods}

Clinical specimens. All experimental procedures were approved by the Ethics Committee of the Second Xiangya Hospital of 
Central South University (ID of ethics document: 2014-S021). Written informed consent was obtained from each patient prior to enrollment. The malignant glioma tissues were obtained from a total of 48 patients with gliomas, including 24 males and 24 females, who underwent surgery at the Second Xiangya Hospital of Central South University (Changsha, China). The mean patient age was 40.5 years (range, 26-71 years). At the time of diagnosis, 23 patients had low-grade (grades I-II) glioma, whereas the other 25 patients had high-grade (grades III-IV) glioma. Adjacent normal brain tissues were obtained from 14 of these patients, including 7 males and 7 females, and the mean patient age was 39.2 years (range, $28-68$ years).

Cell culture. Human glioma cell lines (U-118 MG, LN-18, SW1783 and SW1088) were obtained from the American Type Culture Collection (ATCC; Baltimore, MD, USA), and normal human astrocytes (NHA) were obtained from Lonza (Allendale, NJ, USA). All the cells were cultured in Dulbecco's modified Eagle's medium (DMEM) supplemented with $10 \%$ fetal bovine serum (FBS) (both from Invitrogen Life Technologies, Grand Island, NY, USA), and maintained at $37^{\circ} \mathrm{C}$ with $5 \% \mathrm{CO}_{2}$.

Cell transfection. Cell transfection was performed using Lipofectamine 2000 (Invitrogen Life Technologies) according to the manufacturer's instructions. The pcDNA3.1 vector and the siRNA were obtained from Zhonghong Boyuan Biological Technology Co., Ltd (Shenzhen, China). RACK1-pcDNA3.1 and/or CNTN2 siRNA were transfected into the SW1783 cells, and RACK1 siRNA was transfected into the U-118 MG cells. Briefly, the cells were seeded onto 6-well plates 1 day prior to transfection. The pcDNA3.1 vector, RACK1-pcDNA3.1, scrambled siRNA, RACK1 siRNA, CNTN2 siRNA and Lipofectamine 2000 were diluted in $250-\mu 1$ volumes of Opti-MEM $^{\circledR}$ I reduced-serum medium (Invitrogen Life Technologies) without serum. Subsequently, the plasmid DNA or siRNA were mixed with Lipofectamine 2000 and incubated at room temperature for $20 \mathrm{~min}$ to allow the complexes to form. The complexes were then added to each well.

Cell proliferation assay. For the cell proliferation assay, the cells were seeded onto 96 -well plates at $3 \times 10^{3}$ cells/well. The following day, the cells were transfected with the pcDNA3.1 vector, RACK1-pcDNA3.1, scrambled siRNA and CNTN2 siRNA. At 24, 48 and $72 \mathrm{~h}$ following transfection, cell proliferation was assessed using an MTT cell proliferation and cytotoxicity assay kit (Beyotime, Shanghai, China) according to the manufacturer's instructions. Briefly, $10 \mu \mathrm{l}$ MTT solution $(5 \mathrm{mg} / \mathrm{ml})$ was added to each well and incubated at $37^{\circ} \mathrm{C}$ for $4 \mathrm{~h}$. Subsequently, $100 \mu \mathrm{l}$ dissolving buffer was added to dissolve the formazan crystals. The absorbance at $570 \mathrm{~nm}$ was measured using a microplate reader (BioTek Instruments, Inc., Winooski, VT, USA).

Western blot analysis. Total proteins were extracted from the tissues and cells using ice-cold lysis buffer $[100 \mathrm{mM}$ Tris- $\mathrm{HCl}$ (pH 7.4), $150 \mathrm{mM} \mathrm{NaCl}, 2$ mM DTT, $1 \%$ Triton X-100, $100 \mathrm{mM}$ sodium orthovanadate and $1 \mathrm{mM}$ PMSF]. The protein concentration was determined using a Bradford Protein assay kit (Solarbio, Beijing, China). Equal amounts of protein lysates $(50 \mu \mathrm{g})$ were separated by SDS-PAGE, and the proteins were transferred onto PVDF membranes (Millipore, Billerica, MA, USA). After blocking with 5\% bovine serum albumin (Amresco LLC, Solon, $\mathrm{OH}, \mathrm{USA}$ ), the membranes were washed with TBST 3 times, and incubated with primary antibodies, namely rabbit polyclonal antibody to RACK1 (1:800; ab62735), rabbit polyclonal antibody to CNTN2 (1:400; ab68994), rabbit polyclonal antibody to epidermal growth factor receptor (EGFR; 1:800; ab2430), rabbit polyclonal antibody to platelet-derived growth factor receptor, $\alpha$ polypeptide (PDGFR $\alpha$; 1:500; ab65258), and mouse monoclonal antibody to $\beta$-actin $(1: 2,000 ;$ ab6276) (all from Abcam, Cambridge, MA, USA), mouse monoclonal antibody to Ras (1:500; sc-166691), rabbit polyclonal antibody to glial fibrillary acidic protein (GFAP) (1:400; sc-9065) (all from Santa Cruz Biotechnology, Inc., Santa Cruz, CA, USA), rabbit polyclonal antibody to CD133 (1:400; EAP0023; Elabscience, Hubei, China), rabbit monoclonal antibody to ERK1/2 (1:400; \#4695), rabbit monoclonal antibody to p-ERK1/2 (Thr202/Tyr204; 1:400; \#4377) (Cell Signaling Technology, Inc., Beverly, MA, USA) at $4^{\circ} \mathrm{C}$ overnight, followed by incubation with HRP-conjugated secondary antibodies, namely goat anti-rabbit IgG $\operatorname{HRP}(1: 2,000$; ab6721) and rabbit anti-mouse IgG HRP (1:2,000; ab6728) (both from Abcam) at $37^{\circ} \mathrm{C}$ for $1 \mathrm{~h}$. The immunocomplexes were visualized by enhanced chemiluminescence (Western Blotting Luminol reagent; Santa Cruz Biotechnology, Inc.).

Co-immunoprecipitation assay. For co-immunoprecipitation (co-IP) assay, the cells were extracted using immunoprecipitation lysis buffer (Beyotime), and the supernatants were incubated with $2 \mu \mathrm{g}$ anti-CNTN2 antibody or normal $\operatorname{IgG}$ at $4^{\circ} \mathrm{C}$ overnight. A total of $20 \mu \mathrm{l}$ recombinant fusion protein $\mathrm{A}$ and protein $\mathrm{G}$ agarose (Sigma, St. Louis, MO, USA) was then added followed by incubation at $4^{\circ} \mathrm{C}$ for $3 \mathrm{~h}$. Following centrifugation at $1,000 \mathrm{~g}$ for $5 \mathrm{~min}$ and washing with phosphate-buffered saline (PBS), the immunoprecipitates were collected and subjected to SDS-PAGE.

Statistical analysis. Quantitative data are expressed as the means \pm standard deviation. The two-tailed Student's t-test was performed to analyze the difference between 2 groups using SPSS 19.0 software (SPPS, Inc., Chicago, IL, USA). A P-value $<0.05$ was considered to indicate a statistically significant difference.

\section{Results}

Expression of RACK1 and CNTN2 in human glioma tissues. The expression of RACK1 and CNTN2 in normal brain tissues and glioma tissues was examined by western blot analysis. As shown in Fig. 1, the protein expression of both RACK1 and CNTN2 was significantly higher in the glioma tissues [RACK1, 0.5 \pm 0.11 (low grade) and $0.81 \pm 0.14$ (high grade); CNTN2, $0.52 \pm 0.10$ (low grade) and $0.86 \pm 0.15$ (high grade)] than that in the normal brain tissues (RACK1, 0.23 \pm 0.04 ; CNTN2, 0.31 \pm 0.05 ). Furthermore, it was revealed that the protein expression of RACK1 and CNTN2 in the high-grade glioma tissues was significantly higher than that in the low-grade glioma tissues $(\mathrm{P}<0.05)$.

Expression of RACK1 and CNTN2 in human glioma cell lines. The protein expression of RACK1 and CNTN2 was exam- 

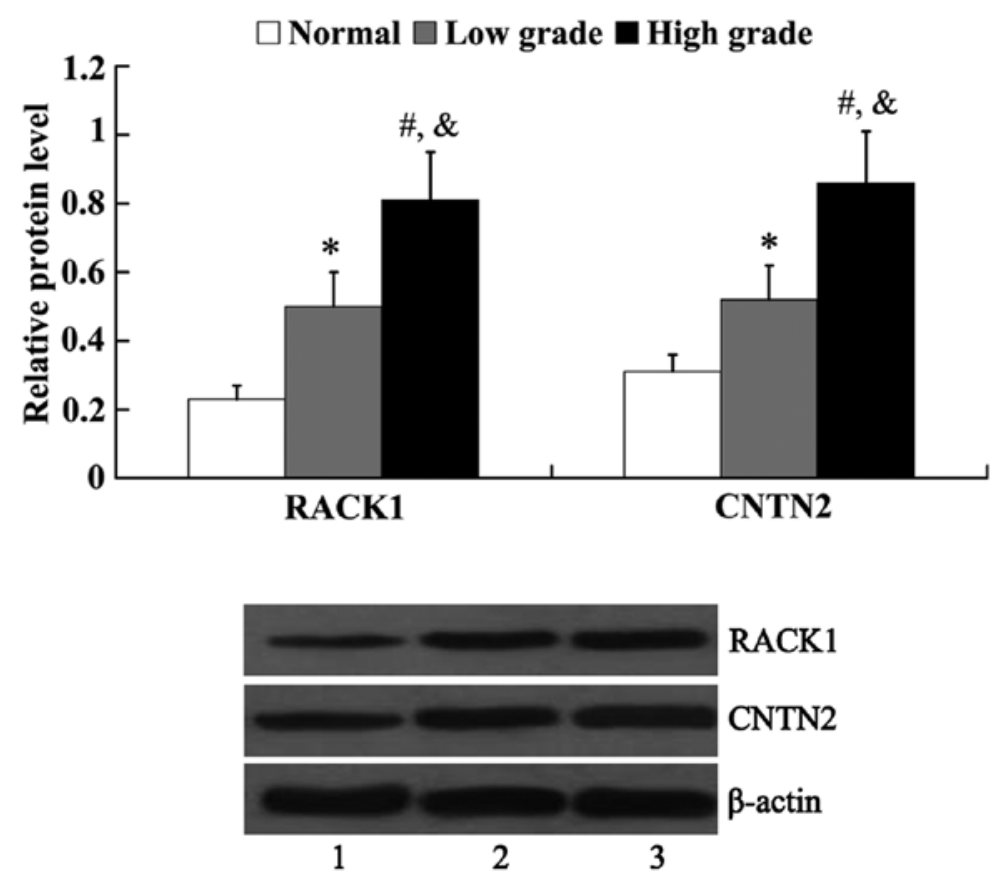

Figure 1. Relative protein levels of receptor for activated C kinase 1 (RACK1) and contactin-2 (CNTN2) in normal human brain tissues and glioma tissues. $\beta$-actin was used as an internal control. Lane 1, normal; lane 2 , low grade; lane 3 , high grade. ${ }^{~} \mathrm{P}<0.05$ and ${ }^{\#} \mathrm{P}<0.01$ compared with the normal brain tissues; ${ }^{\&} \mathrm{P}<0.05$ compared with the low-grade glioma tissues.
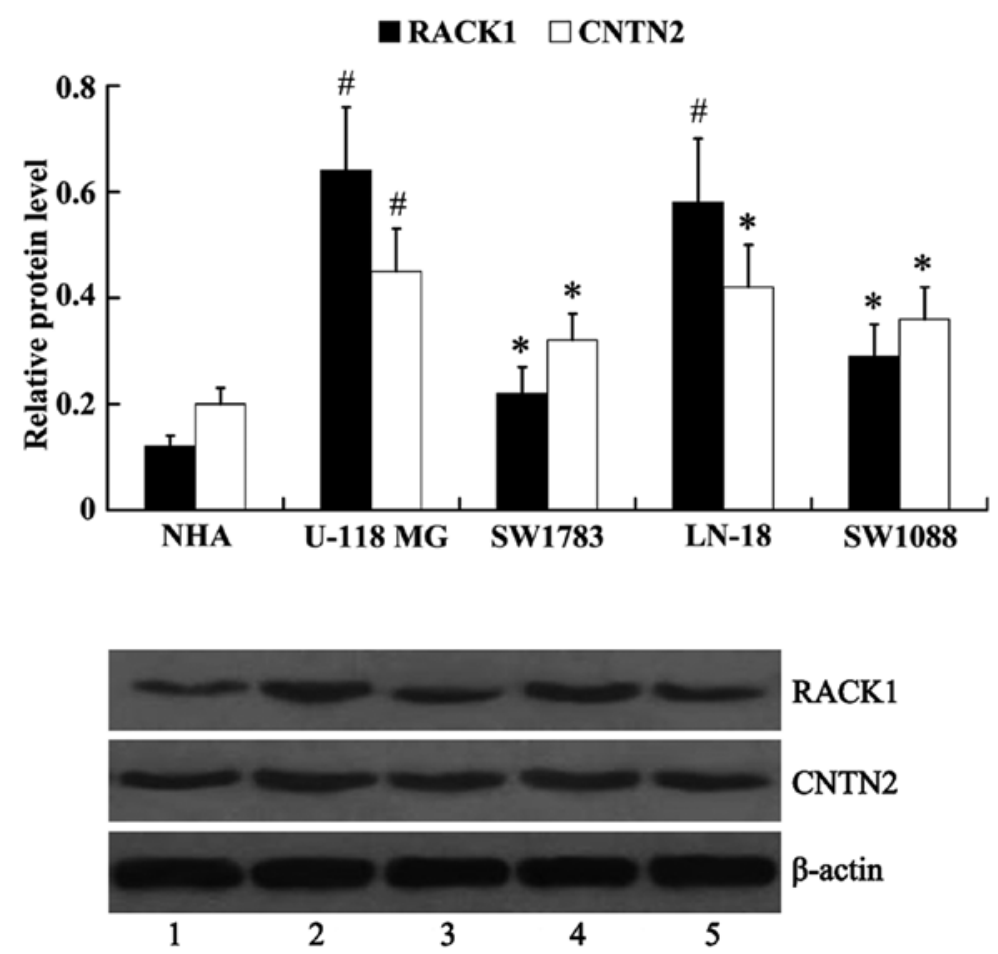

Figure 2. Relative protein levels of receptor for activated C kinase 1 (RACK1) and contactin-2 (CNTN2) in normal human astrocytes (NHA) and glioma cell lines (U-118 MG, SW1783, LN-18 and SW1088). $\beta$-actin was used as an internal control. Lanes 1-5 represent the NHA, U-118 MG, SW1783, LN-18 and SW1088 cells, respectively. ${ }^{*} \mathrm{P}<0.05$ and ${ }^{\#} \mathrm{P}<0.01$ compared with NHA.

ined in human glioma cell lines (U-118 MG, LN-18, SW1783 and SW1088). Normal human astrocytes (NHA) were used as controls. It was noted that, compared with the NHA cells (RACK1, $0.12 \pm 0.02 ; \mathrm{CNTN} 2,0.20 \pm 0.03$ ), the protein expression levels of RACK1 and CNTN2 were upregulated in human glioma cells [RACK1: $0.64 \pm 0.12$ (U-118 MG), $0.22 \pm 0.05$ (SW1783), $0.58 \pm 0.12$ (LN-18), $0.29 \pm 0.06$ (SW1088); CNTN2: $0.45 \pm 0.08$ (U-118 MG), 0.32 \pm 0.05 (SW1783), 0.42 \pm 0.08 (LN-18), $0.36 \pm 0.06$ (SW1088)], with the highest expression noted in the U-118 MG cells, and lowest in the SW1783 cells (Fig. 2). 


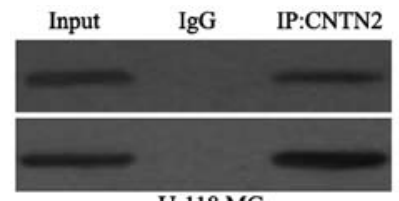

U-118 MG

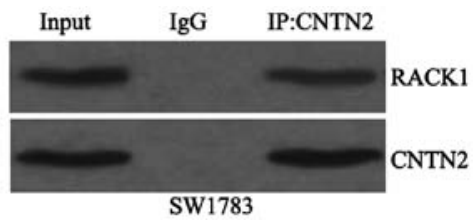

SW1783
Figure 3. Co-immunoprecipitation assay of receptor for activated $\mathrm{C}$ kinase 1(RACK1) and contactin-2 (CNTN2) CNTN2 in U-118 MG and SW1783 cells. Input lanes represent the total cell lysates. IgG was used as a control.

RACK1 interacts with and regulates the expression of CNTN2. To determine whether RACK1 interacts with CNTN2, we examined RACK1 expression after cell lysates were immunoprecipitated with an anti-CNTN2 antibody. As shown in Fig. 3, RACK1 protein was detected after immunoprecipitation of endogenous CNTN2 from cell lysates.

The SW1783 cells demonstrated the lowest protein expression of RACK1; thus, we transfected RACK1-pcDNA3.1 into the SW1783 cells to induce the overexpression of RACK1. RACK1 siRNA was transfected into the U-118 MG cells to knockdown endogenous RACK1, as the U-118 MG cells exhibited the highest expression of RACK1. Subsequently, the protein expression of RACK1 and CNTN2 was examined. The results from western blot analysis revealed that RACK1 protein expression was significantly increased in the SW1783 cells following transfection with RACK1-pcDNA3.1 (0.89 \pm 0.17 vs. $0.20 \pm 0.04$, $\mathrm{P}<0.01$ ); however, RACK1 protein expression was decreased in the U-118 MG cells following transfection with RACK1 siRNA $(0.10 \pm 0.02$ vs. $0.65 \pm 0.12, \mathrm{P}<0.01)$. The overexpression of RACK1 led to the increased expression of CNTN2 $(0.62 \pm 0.11$ vs. $0.30 \pm 0.05, \mathrm{P}<0.05)$, whereas the knockdown of RACK1 exerted an inhibitory effect on the expression of CNTN2 (0.21 \pm 0.04 vs. $0.48 \pm 0.09, \mathrm{P}<0.01)$ (Fig. 4).

CNTN2 mediates the effects of RACK1 on cell proliferation. RACK1-pcDNA3.1 and/or CNTN2 siRNA were transfected into the SW1783 cells, and the protein expression levels of RACK1 and CNTN2 were then examined. As shown in Fig. 5, the expression of CNTN2 was significantly decreased in the cells following transfection with CNTN2 siRNA $(0.31 \pm 0.05$ vs $.0 .08 \pm 0.02, \mathrm{P}<0.01 ; 0.60 \pm 0.11$ vs. $0.18 \pm 0.04, \mathrm{P}<0.01)$. However, the expression of RACK1 was not markedly altered by transfection with CNTN2 siRNA ( $0.21 \pm 0.04$ vs. $0.19 \pm 0.04, \mathrm{P}>0.05$; $0.92 \pm 0.19$ vs. $0.89 \pm 0.18, \mathrm{P}>0.05$ ).

An MTT assay was performed to determine cell proliferation ability. As shown in Fig. 6, cell viability was inhibited in the cells following transfection with CNTN2 siRNA for 48 and $72 \mathrm{~h}$ $(\mathrm{P}<0.05)$. RACK1-pcDNA3.1 promoted cell proliferation (48 h, $175 \pm 16 \%$ vs. $216 \pm 18 \%, \mathrm{P}<0.05 ; 72 \mathrm{~h}, 219 \pm 18 \%$ vs. $266 \pm 20 \%$, $\mathrm{P}<0.05)$; however, this effect was abolished by the knockdown of CNTN2 ( 48 h, $216 \pm 18 \%$ vs. $170 \pm 17 \%, \mathrm{P}<0.05 ; 72 \mathrm{~h}$, $266 \pm 20 \%$ vs. $213 \pm 18 \%, \mathrm{P}<0.05)$.

CNTN2 mediates the effect of RACK1 on cell differentiation. The expression of GFAP and CD133 was examined by western blot analysis to determine cell differentiation. We found that RACK1 inhibited cell differentiation, as evidenced by the markedly decreased expression of GFAP and the increased expression of CD133 in the cells following transfection with
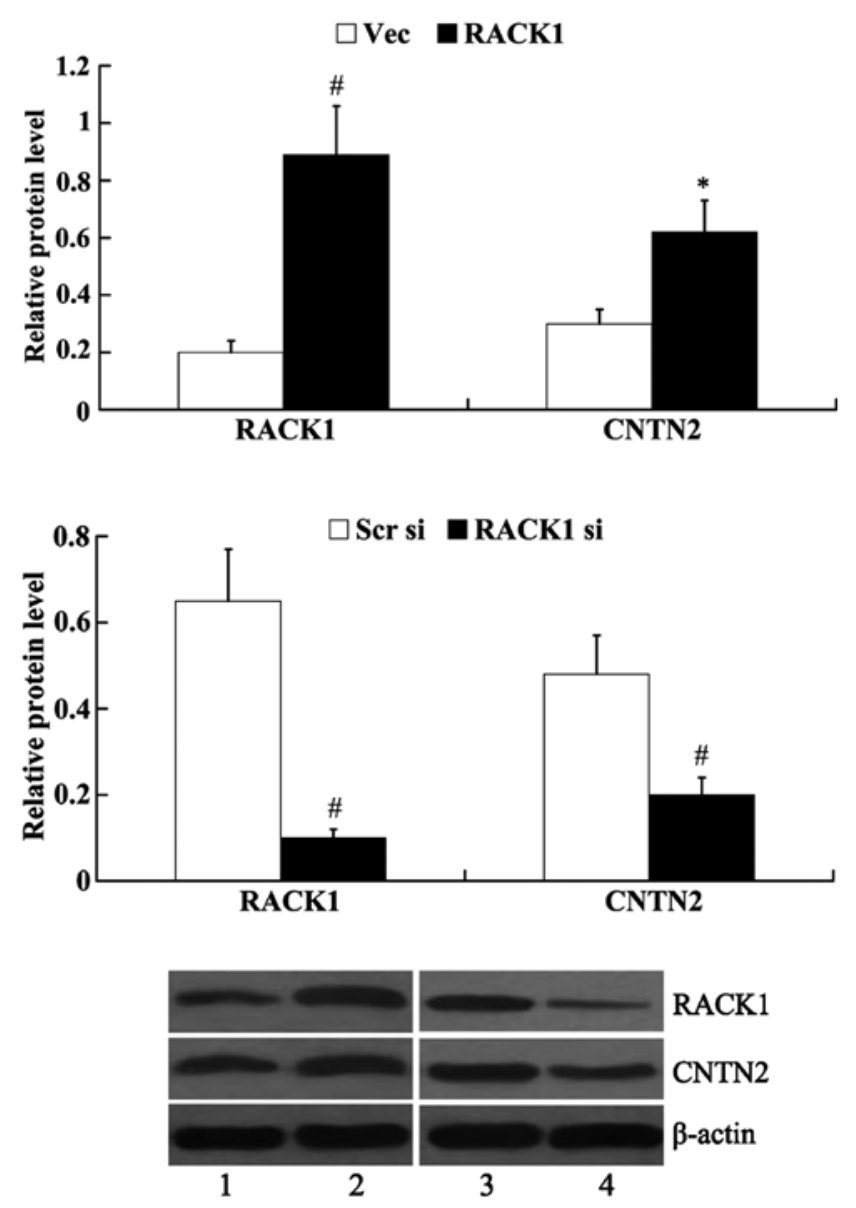

Figure 4. Relative protein levels of receptor for activated C kinase 1 (RACK1) and contactin-2 (CNTN2) in SW1783 cells following transfection with RACK1pcDNA3.1 and in U-118 MG cells following transfection with RACK1 siRNA (RACK1 si). $\beta$-actin was used as an internal control. Lane 1, pcDNA3.1 vector (Vec); lane 2, RACK1-pcDNA3.1; lane 3, scramble siRNA (Scr si); lane 4, RACK1 siRNA. " $\mathrm{P}<0.05$ and ${ }^{\#} \mathrm{P}<0.01$ compared with the Vec group or Scr si group.

RACK1-pcDNA3.1 (GFAP, $0.25 \pm 0.04$ vs. $0.15 \pm 0.03, \mathrm{P}<0.05$; CD133, $0.20 \pm 0.04$ vs. $0.45 \pm 0.08, \mathrm{P}<0.01)$. The knockdown of CNTN2 attenuated the effects of RACK1 on cell differentiation, as the expression of GFAP was significantly upregulated and CD133 was significantly downregulated following transfection with CNTN2 siRNA (GFAP, $0.15 \pm 0.03$ vs. $0.28 \pm 0.05$, $\mathrm{P}<0.05$; $\mathrm{CD} 133,0.45 \pm 0.08$ vs. $0.18 \pm 0.03, \mathrm{P}<0.01$ ) (Fig. 7).

CNTN2 mediates the effect of RACK1 on RTK/Ras/MAPK signaling. In order to examine the activation of RTK/Ras/ MAPK signaling, the expression of molecules in this signaling pathway, including EGFR, PDGFR $\alpha$, Ras and phosphorylated (p-)ERK1/2 were examined by western blot analysis. As shown in Fig. 8, the RTK/Ras/MAPK signaling pathway was activated by RACK1, accompanied by the increased expression of EGFR, PDGFR $\alpha$, Ras and p-ERK $1 / 2$ in the cells overexpressing RACK1 (EGFR, $0.49 \pm 0.07$ vs. $0.82 \pm 0.15, \mathrm{P}<0.05$; PDGFR $\alpha$, $0.32 \pm 0.05$ vs. $0.60 \pm 0.10, \mathrm{P}<0.05$; Ras, $0.31 \pm 0.05$ vs. $0.55 \pm 0.09$, $\mathrm{P}<0.05 ; \mathrm{p}-\mathrm{ERK} 1 / 2,0.40 \pm 0.08$ vs. $0.75 \pm 0.15, \mathrm{P}<0.05$ ). However, the activation of the RTK/Ras/MAPK signaling pathway was abolished by the knockdown of CNTN2 (EGFR, $0.82 \pm 0.15$ vs. $0.45 \pm 0.08, \mathrm{P}<0.05 ; \mathrm{PDGFR} \alpha, 0.60 \pm 0.10$ vs. $0.28 \pm 0.07, \mathrm{P}<0.05$; 

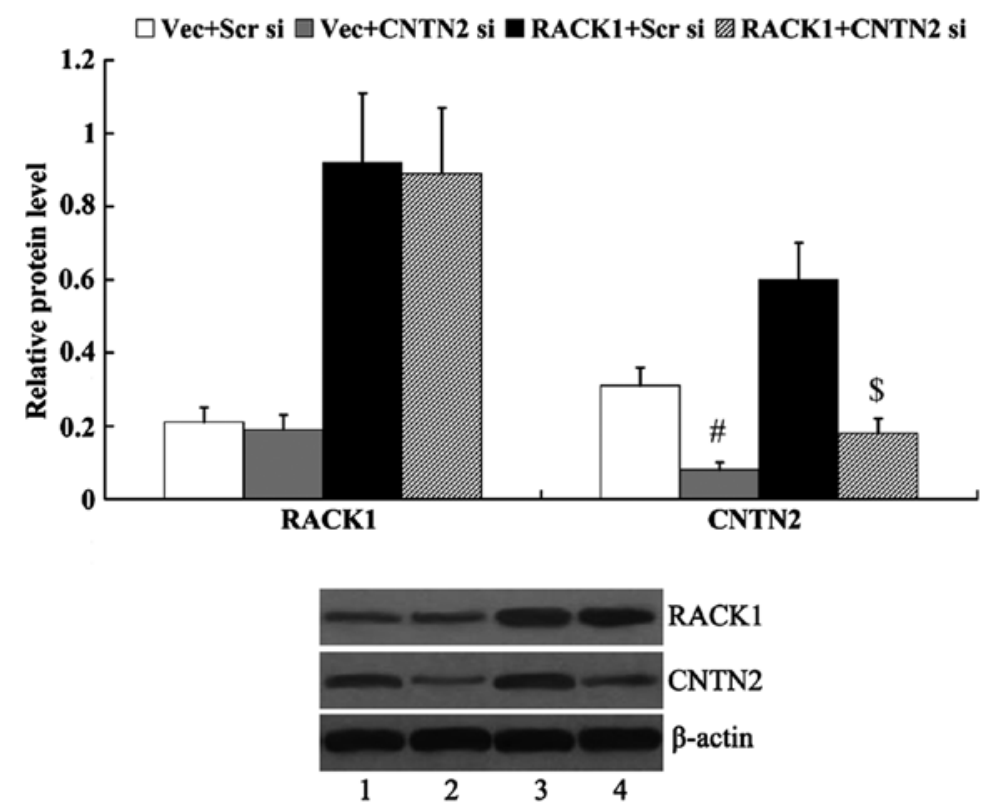

Figure 5. Relative protein levels of receptor for activated C kinase 1 (RACK1) and contactin-2 (CNTN2) in cells following transfection with RACK1-pcDNA3.1 and CNTN2 siRNA. $\beta$-actin was used as an internal control. Lane 1, Vec + Scr si; lane 2, Vec + CNTN2 si; lane 3, RACK1 + Scr si; lane 4, RACK1 + CNTN2 siRNA. ${ }^{\text {"P }}<0.01$ compared with the Vec + Scr si group; ${ }^{\$} \mathrm{P}<0.01$ compared with the RACK1 + Scr si group. Vec, pcDNA3.1 vector; Scr si, scrambled siRNA.

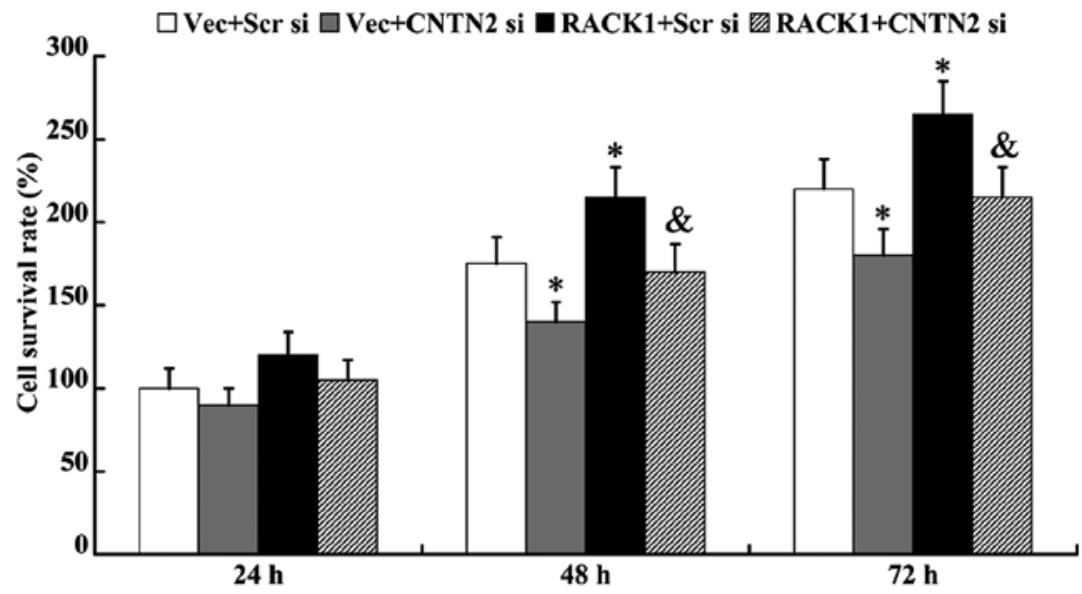

Figure 6. Cell proliferation following transfection with receptor for activated C kinase 1 (RACK1)-pcDNA3.1 and contactin-2 (CNTN2) siRNA. "P<0.05 compared with the Vec + Scr si group; ${ }^{\&} \mathrm{P}<0.05$ compared with the RACK1 + Scr si group. Vec, pcDNA3.1 vector; Scr si, scrambled siRNA.

Ras, $0.55 \pm 0.09$ vs. $0.30 \pm 0.05, \mathrm{P}<0.05 ; \mathrm{p}$-Erk $1 / 2,0.75 \pm 0.15$ vs. $0.38 \pm 0.08, \mathrm{P}<0.05)$.

\section{Discussion}

In the present study, we found that the protein expression levels of RACK1 and CNTN2 were higher in high-grade glioma tissues and cells, and lower in low-grade glioma tissues and cells. RACK1 is a scaffold protein that physically or functionally interacts with numerous proteins $(8,21-23)$. CNTN2 is a cell adhesion protein that is expressed mainly in axons or regenerating neurons (26). It is involved in the differentiation of cerebellar neurons (27), and is linked to the highest degree in the oligodendroglioma protein-protein interaction network (20). However, to the best of our knowledge, this is the first study to examine whether RACK1 interacts with CNTN2.
To examine the association between RACK1 and CNTN2, a co-immunoprecipitation assay was performed, and the results revealed that RACK1 interacts with CNTN2. Furthermore, the results from western blot analysis demonstrated that RACK1 upregulated the expression of CNTN2. However, the knockdown of CNTN2 did not have a marked effect on the expression of RACK1. These data suggest that CNTN2 is a downstream effector of RACK1.

It has been suggested in previous studies that the dysregulation of RACK1 is involved in a variety of cancers, such as esophageal squamous cell carcinoma, lung cancer and ovarian cancer (10-13). In the study by Peng et al, it was demonstrated that the enforced downregulation of RACK1 inhibited tumor xenograft growth in nude mice, and inhibited the proliferation and invasion of human glioma cells in vitro (14). In the present study, we examined the effects of RACK1 on glioma 

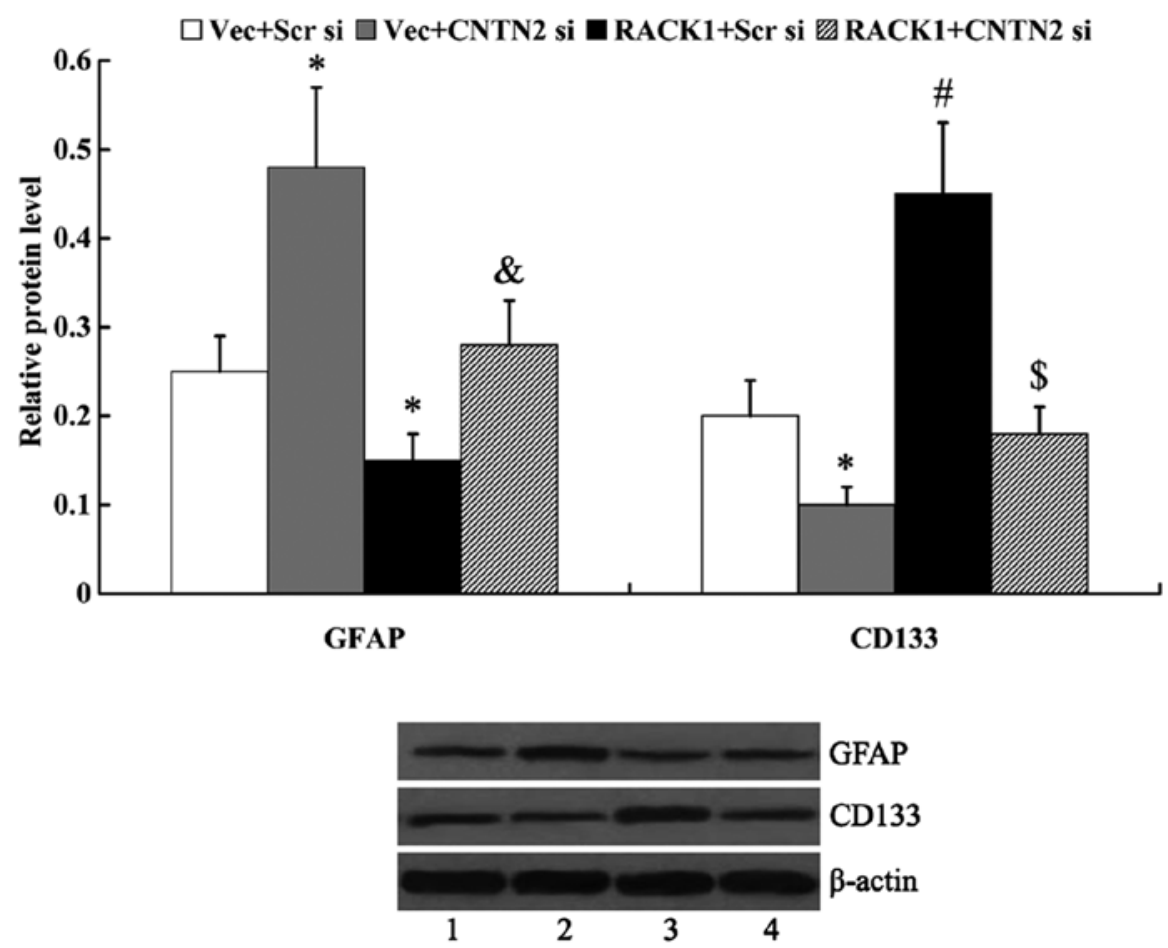

Figure 7. Relative protein levels of cell differentiation markers glial fibrillary acidic protein (GFAP) and CD133 in cells following transfection with receptor for activated C kinase 1 (RACK1)-pcDNA3.1 and contactin-2 (CNTN2) siRNA. $\beta$-actin was used as an internal control. Lane 1, Vec + Scr si; lane 2, Vec + CNTN2 siRNA (si); lane 3, RACK1 + Scr si; lane 4, RACK1 + CNTN2 si. " $\mathrm{P}<0.05$ and ${ }^{\sharp} \mathrm{P}<0.01$ compared with the Vec + Scr si group; ${ }^{\text {\& }} \mathrm{P}<0.05$ and ${ }^{\$} \mathrm{P}<0.01$ compared with the RACK1 + Scr si group. Vec, pcDNA3.1 vector; Scr si, scrambled siRNA.

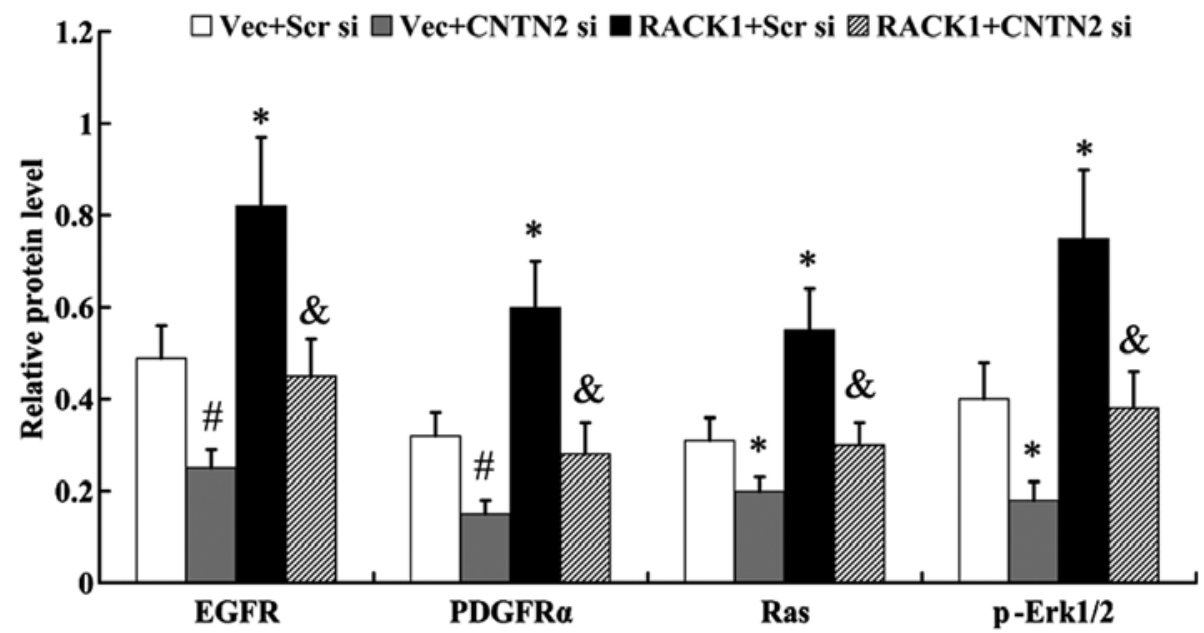

Figure 8. Relative protein levels of molecules in receptor tyrosine kinase (RTK)/Ras/mitogen-activated protein kinase (MAPK) signaling in cells following transfection with the receptor for activated C kinase 1 (RACK1)-pcDNA3.1 and contactin-2 (CNTN2) siRNA. $\beta$-actin was used as an internal control for the expression level of epidermal growth factor receptor (EGFR), platelet-derived growth factor receptor, $\alpha$ polypeptide (PDGFR $\alpha$ ) and Ras. The relative expression level of phosphorylated extracellular signal-regulated protein kinase (p-ERK)1/2 were normalized to total ERK1/2. Lane 1, pcDNA3.1 Vec + Scr si; lane 2, Vec + CNTN2 si; lane 3, RACK1 + Scr si; lane 4, RACK1 + CNTN2 si. " $\mathrm{P}<0.05$ and ${ }^{\#} \mathrm{P}<0.01$ compared with the Vec + Scr si group; ${ }^{\circledR} \mathrm{P}<0.05$ compared with the RACK1 + Scr si group. Vec, vector; Scr si, scrambled siRNA.

cell differentiation. We found that RACK1 had an inhibitory effect on the differentiation of glioma cells, and we noted the decreased expression of the differentiation marker, GFAP, and the increased expression of the glioma stem cell self-renewal marker, CD133, in the cells overexpressing RACK1. Similar to the effects of RACK1, CNTN2 also inhibited glioma cell differentiation, and the effects of RACK1 on glioma cell differentiation were abolished by the knockdown of CNTN2.
RTK signaling pathways control a number of biological processes, including cell proliferation, survival, differentiation and morphogenesis $(28,29)$. EGFR and PDGFR are two important RTKs. The increase in RTK activity initiates signaling through the conserved Ras/MAPK cassette, thus regulating the development of glioblastoma multiforme and other cancers $(30,31)$. In the present study, we found that the RTK/ Ras/MAPK signaling pathway was activated by RACK1 and 
CNTN2 in glioma cells. In addition, the knockdown of CNTN2 attenuated the effects of RACK1 on the activation of the RTK/ Ras/MAPK signaling pathway.

Taken together, our findings firstly demonstrated that RACK1 interacts with and regulates CNTN2, and the effects of RACK1 on glioma cell growth and differentiation were mediated by CNTN2. Thus, we suggest that the RACK1/CNTN2/RTK/ Ras/MAPK axis exists in glioma cells and this axis may be a potential target in the treatment of gliomas.

\section{References}

1. Katsetos CD, Dráberová E, Smejkalová B, Reddy G, Bertrand L, de Chadarévian JP, Legido A, Nissanov J, Baas PW and Dráber P Class III beta-tubulin and gamma-tubulin are co-expressed and form complexes in human glioblastoma cells. Neurochem Res 32: 1387-1398, 2007.

2. Arko L, Katsyv I, Park GE, Luan WP and Park JK: Experimental approaches for the treatment of malignant gliomas. Pharmacol Ther 128: 1-36, 2010.

3. Yacoub A, Hamed HA, Allegood J, Mitchell C, Spiegel S, Lesniak MS, Ogretmen B, Dash R, Sarkar D, Broaddus WC, et al: PERK-dependent regulation of ceramide synthase 6 and thioredoxin play a key role in mda-7/IL-24-induced killing of primary human glioblastoma multiforme cells. Cancer Res 70: 1120-1129, 2010

4. Hamed HA, Yacoub A, Park MA, Eulitt PJ, Dash R, Sarkar D, Dmitriev IP, Lesniak MS, Shah K, Grant S, et al: Inhibition of multiple protective signaling pathways and Ad.5/3 delivery enhances mda-7/IL-24 therapy of malignant glioma. Mol Ther 18: 1130-1142, 2010.

5. Yu H, Park J, Lee J, Choi K and Choi C: Constitutive expression of MAP kinase phosphatase-1 confers multi-drug resistance in human glioblastoma cells. Cancer Res Treat 44: 195-201, 2012.

6. Xing WJ, Zou Y, Han QL, Dong YC, Deng ZL, Lv XH, Jiang T and Ren H: Effects of epidermal growth factor receptor and phosphatase and tensin homologue gene expression on the inhibition of U87MG glioblastoma cell proliferation induced by protein kinase inhibitors. Clin Exp Pharmacol Physiol 40: 13-21, 2013.

7. Gandin V, Senft D, Topisirovic I and Ronai ZA: RACK1 Function in Cell Motility and Protein Synthesis. Genes Cancer 4: 369-377, 2013.

8. Adams DR, Ron D and Kiely PA: RACK1, a multifaceted scaffolding protein: structure and function. Cell Commun Signal 9: 22, 2011.

9. McCahill A, Warwicker J, Bolger GB, Houslay MD and Yarwood SJ: The RACK1 scaffold protein: a dynamic cog in cell response mechanisms. Mol Pharmacol 62: 1261-1273, 2002.

10. Li JJ and Xie D: RACK1, a versatile hub in cancer. Oncogene 34: 1890-1898, 2015.

11. Wang N, Liu F, Cao F, Jia Y, Wang J, Ma W, Tan B, Wang K, Song $\mathrm{Q}$ and Cheng Y: RACK1 predicts poor prognosis and regulates progression of esophageal squamous cell carcinoma through its epithelial-mesenchymal transition. Cancer Biol Ther 16: 528-540, 2015 .

12. Choi YY, Lee SY, Lee WK, Jeon HS, Lee EB, Lee HC, Choi JE, Kang HG, Lee EJ, Bae EY, et al: RACK1 is a candidate gene associated with the prognosis of patients with early stage non-small cell lung cancer. Oncotarget 6: 4451-4466, 2015.

13. Lin Y, Cui M, Teng H, Wang F, Yu W and Xu T: Silencing the receptor of activated C-kinase 1 (RACK1) suppresses tumorigenicity in epithelial ovarian cancer in vitro and in vivo. Int $\mathrm{J}$ Oncol 44: 1252-1258, 2014.
14. Peng R, Jiang B, Ma J, Ma Z, Wan X, Liu H, Chen Z, Cheng Q and Chen R: Forced downregulation of RACK1 inhibits glioma development by suppressing Src/Akt signaling activity. Oncol Rep 30: 2195-2202, 2013

15. Su J, Xu J and Zhang S: RACK1, scaffolding a heterotrimeric $\mathrm{G}$ protein and a MAPK cascade. Trends Plant Sci 20: 405-407, 2015.

16. Shi S, Deng YZ, Zhao JS, Ji XD, Shi J, Feng YX, Li G, Li JJ, Zhu D, Koeffler HP, et al: RACK1 promotes non-small-cell lung cancer tumorigenicity through activating sonic hedgehog signaling pathway. J Biol Chem 287: 7845-7858, 2012.

17. Chen Y, Wang L, Xu H, Liu X and Zhao Y: Exome capture sequencing reveals new insights into hepatitis B virus-induced hepatocellular carcinoma at the early stage of tumorigenesis. Oncol Rep 30: 1906-1912, 2013.

18. Adair SJ, Carr TM, Fink MJ, Slingluff CL Jr and Hogan KT: The TAG family of cancer/testis antigens is widely expressed in a variety of malignancies and gives rise to HLA-A2-restricted epitopes. J Immunother 31: 7-17, 2008.

19. Rickman DS, Tyagi R, Zhu XX, Bobek MP, Song S, Blaivas M, Misek DE, Israel MA, Kurnit DM, Ross DA, et al: The gene for the axonal cell adhesion molecule TAX-1 is amplified and aberrantly expressed in malignant gliomas. Cancer Res 61: 2162-2168, 2001

20. Yu F and Fu WM: Identification of differential splicing genes in gliomas using exon expression profiling. Mol Med Rep 11: 843-850, 2015

21. Birikh KR, Sklan EH, Shoham S and Soreq H: Interaction of 'readthrough' acetylcholinesterase with RACK1 and PKCbeta II correlates with intensified fear-induced conflict behavior. Proc Natl Acad Sci USA 100: 283-288, 2003.

22. Wang CC, Lo HF, Lin SY and Chen H: RACK1 (receptor for activated C-kinase 1) interacts with FBW2 (F-box and WD-repeat domain-containing 2) to up-regulate GCM1 (glial cell missing 1) stability and placental cell migration and invasion. Biochem J 453: 201-208, 2013.

23. Kundu N, Dozier U, Deslandes L, Somssich IE and Ullah H: Arabidopsis scaffold protein RACK1A interacts with diverse environmental stress and photosynthesis related proteins. Plant Signal Behav 8: e24012, 2013.

24. Wee B, Charles N and Holland EC: Animal models to study cancer-initiating cells from glioblastoma. Front Biosci (Landmark Ed) 16: 2243-2258, 2011.

25. Akhavan D, Cloughesy TF and Mischel PS: mTOR signaling in glioblastoma: lessons learned from bench to bedside. Neuro Oncol 12: 882-889, 2010.

26. Brümmendorf T and Rathjen FG: Axonal glycoproteins with immunoglobulin- and fibronectin type III-related domains in vertebrates: structural features, binding activities, and signal transduction. J Neurochem 61: 1207-1219, 1993.

27. Bizzoca A, Virgintino D, Lorusso L, Buttiglione M, Yoshida L, Polizzi A, Tattoli M, Cagiano R, Rossi F, Kozlov S, et al: Transgenic mice expressing F3/contactin from the TAG-1 promoter exhibit developmentally regulated changes in the differentiation of cerebellar neurons. Development 130: 29-43, 2003.

28. Schlessinger J: Cell signaling by receptor tyrosine kinases. Cell 103: 211-225, 2000

29. Simon MA: Receptor tyrosine kinases: specific outcomes from general signals. Cell 103: 13-15, 2000.

30. Carracedo A, Ma L, Teruya-Feldstein J, Rojo F, Salmena L, Alimonti A, Egia A, Sasaki AT, Thomas G, Kozma SC, et al: Inhibition of mTORC1 leads to MAPK pathway activation through a PI3K-dependent feedback loop in human cancer. J Clin Invest 118: 3065-3074, 2008.

31. Wan X, Harkavy B, Shen N, Grohar P and Helman LJ: Rapamycin induces feedback activation of Akt signaling through an IGF-1R-dependent mechanism. Oncogene 26: 1932-1940, 2007. 